\title{
A New Form of Intraoral Delivery of Antifungal Drugs for the Treatment of Denture-Induced Oral Candidosis
}

Wala M. Amin ${ }^{a}$

Muna H. Al-Alib

Nesreen A. Salimb

Sandra K. Al-Tarawneh ${ }^{b}$

\section{ABSTRACT}

Objectives: To monitor the release of the antifungal drugs Fluconazole, Chlorhexidine and a combination of the two from an auto-polymerized poly (methyl methacrylate) (PMMA) denture base resin; and to investigate the effect of the released drugs upon the growth of Candida albicans.

Methods: A high performance liquid chromatography-Ultra violet (HPLC-UV) method was used in the analysis of the released drugs into distilled water from PMMA discs doped with the antifungal drugs Fluconazole $(10 \%)$, Chlorhexidine $(10 \%)$ and a combination of the two drugs ( $5 \%$ each). The antifungal efficacy of the released drugs was monitored, microbiologically, employing "well" technique on a Saborauds culture medium inoculated with a resistant strain of Candida albicans.

Results: It was shown that Fluconazole, Chlorhexidine and the combination of the two drugs can be successfully incorporated with PMMA. It was found that the drugs leach steadily out of the PMMA resin into distilled water at mouth temperature and that sustained drug release continued throughout the 28 days test period. It was also shown that the released drugs demonstrated an antifungal activity against the resistant Candida albicans and this was most remarkable in the combined drugs samples.

Conclusions: The findings of this investigation have a clinical value in terms of their significant contribution to the treatment of fungal infections of the oral cavity. The sustained release of antifungal drugs from the PMMA resin clearly constitutes a new dosage form of these drugs via the poly (methyl methacrylate) delivery system. (Eur J Dent 2009;3:257-266)

Key words: Fluconazole; Chlorhexidine; Synergism; Auto-polymerized PMMA; Eluates; Antifungal drug; HPLC-UV; Denture stomatitis; Candida albicans.

- a Professor of Prosthodontics and Biomaterials, Dept. of Prosthetic Dentistry, Faculty of Dentistry, University of Jordan, Jordan.

b Teaching assistant and clinical instructor, Faculty of Dentistry, University of Jordan, Jordan.

- Corresponding author: Professor Wala M. Amin P.0.Box: 13455, Amman 11942, Jordan

Phone: +96265355000 , Ext. 23601

E-mail: profwaladgmail.com

\section{INTRODUCTION}

The Candida-associated denture stomatitis is a common condition characterized by generalized inflammation of the palatal mucosa covered by the denture. ${ }^{1,2}$ It is a harmless form of oral candidiasis and is associated with a quantitative increase of yeasts on the mucosa and the denture's fit surface. ${ }^{2}$ Usually a mixture of Candida species such 
as Candida albicans, Candida tropicals, Candida krusei, Candida guilliermondii, Candida parapsilosis, Candida glabrata can be isolated from oral candidal lesions. Candida albicans, however, has been claimed to be the principal pathogen that has a main role in the development of oral candidosis. ${ }^{3}$ The presence of numerous yeasts may give rise to spreading to the angles of the mouth, the tongue, the pharynx, and the alimentary and respiratory tracts. ${ }^{4}$ The lesion may heal partially after topical antifungal treatment ${ }^{4,5}$ but the incidence of relapse is, however, very high. ${ }^{6-8}$

Although denture induced candidosis could be treated by methods targeted towards the oral mucosa, other treatment modalities are directed toward the denture base. A number of effective antifungal agents have been administered, either topically or systemically, for management of oral candidosis. ${ }^{9}$ Amphotricin B and nystatin are common topical antifungal agents, whereas azoles such as fluconazole and ketoconazole are available as systemic antifungal treatment. ${ }^{10}$ Significant resistance in yeasts has been reported in isolates from patients with prolonged neutropenia, ${ }^{11}$ and in HIV patients. ${ }^{12}$

Chlorhexidine gluconate, an antimicrobial agent, has been reported as an active against a broad spectrum of organisms including Candida. ${ }^{13,14}$ Although systemic dosage forms may be effective against mucosal lesions; they offer no cure to the Candida infested tissue surface of dentures. Persistent fungal presence on the denture's fitting surface often leads to cross infection and recurrence of the mucosal lesions. Candida albicans has been found on both hard denture base acrylic resins and silicon-based resilient liner materials in vivo and in vitro. ${ }^{15}$

Several researches investigated the feasibility of using drug delivery system by incorporation of antifungal or antimicrobial agents, with denture acrylic resin ${ }^{16}$ or with soft liners. ${ }^{17}$ The idea suggested the use of polymerized acrylic as carriers for drugs orally. ${ }^{18}$ Similarly, soft liners placed in dentures have been used as carriers for antifungal drugs in treating denture stomatitis. ${ }^{19,20}$

It has been found that the release of the drug out of the polymeric carriers continued for more than 100 days. ${ }^{21}$ However, the physical properties of the resin were affected due to the presence of the drug particles, which may dissolve and result in porosity in the acrylic base. ${ }^{22}$
These formulations have been claimed of having less side effects compared with the conventional forms, because of the continual presence of the drug at the site of action, less amount of drug is needed to achieve the therapeutic effect. ${ }^{23}$ They were also believed to be of high benefit for physically or mentally compromised patients. ${ }^{24}$ An example of these formulations, in which sustained topical administration of chlorhexidine via a self-cured polymeric system based on polyethyl methacrylate and tetrahydrofurfuryl methacrylate (PEM/THFM) had been advocated. ${ }^{25}$

The methods of detecting the released antifungal drug varied among investigators; some used spectrophotometric and agar diffusion measurements of the released drug into water; ${ }^{21}$ others employed a proton nuclear magnetic resonance spectroscopy for the same purpose ${ }^{25}$ whereas some researchers used ultraviolet spectrometry in the measurement of drug release. ${ }^{26}$

All previous studies, except one, stopped short of investigating whether the concentration of the released antifungal drug into the surrounding medium was adequate to produce the required role. One study, ${ }^{26}$ however used a turbidimetric measurement of candidal growth after exposure to an experimental drug release device both with and without the antifungal drug. It was found that the drug-containing test samples inhibited candidal growth and survival, in vitro. The number of surviving colony-forming unit $/ \mathrm{ml}$ of culture medium was substantially reduced for the test sample compared to controls.

Nowadays HPLC is used for a wide range of applications and offers significant advantages in the analysis of pharmaceutical formulations and biological fluids. An added advantage is that many detectors used in HPLC are non-destructive, thus facilitating sample recovery. ${ }^{27}$

The present study aimed to formulating a medicated relining polymeric delivery system based on the conventional auto-polymerizing poly (methyl methacrylate) resin doped with $10 \%(\mathrm{w} / \mathrm{w})$ antifungal drug for sustained topical delivery of the drug for the treatment of denture-induced oral candidosis.

The choice of the polymeric system was based on its abundance, ease of handling and cost effectiveness. The room-temperature polymerizing resin version was employed because it has been 
proven that this mode of polymerization does not adversely affect the efficacy of the antifungal drugs. ${ }^{26}$ The employed polymer was intended for use as an indirect relining resin in order to avoid the possible thermal trauma to the denture bearing mucosa that may be caused by the polymerization exotherm.

The antifungal drugs employed in the study were fluconazole (10\%); chlorhexidine (10\%); and a combination of fluconazole and chlorhexidine ( $5 \%$ each). The chosen concentration of the drugs, i.e., $10 \%$, represents the maximum limit that has no discernible effect on the preparation of the polymer samples, ${ }^{26}$ and at the same time it is the limit required to affect antifungal activity over the intended therapeutic period.

The inclusion of fluconazole in the study, despite previous reports ${ }^{11,12}$ that cast doubt on its effectiveness against the azole resistant strains of Candida, was in order to: firstly, provide a microbiological evidence either for or against the reported argument; and secondly, to acquire a base line data essential for comparing the antifungal efficacy of fluconazole-doped samples with those doped with chlorhexidine as well as with samples doped with the combined fluconazole/chlorhexidine antifungal drugs.

\section{MATERIALS AND METHODS}

\section{Sample preparation}

A room temperature-polymerized poly (methyl methacrylate) acrylic resin, a product of (Paladur: powder batch No. 32, liquid batch No. 012290) was employed. The mixing ratio and the conditions for processing and polymerization recommended by the manufacturer were strictly followed. These were $5 \mathrm{~g} / 3 \mathrm{ml}$ polymer powder/ monomer liquid ratio for mixing. Three groups of medicated samples were prepared (Figure 1). The first group was impregnated with $10 \% \mathrm{w} / \mathrm{w}$ Fluconazole powder; the second group with $10 \%$ Chlorhexidine powder; and the third group was impregnated with a combination of Fluconazole $5 \% \mathrm{w} / \mathrm{w}$ powder and Chlorhexidine $5 \% \mathrm{w} / \mathrm{w}$ powder. The drugs were added in the specified ratio to the acrylic resin powder then the mixture of the powders with the liquid monomer were stirred for 15 seconds and left standing for 4 minutes until plastic dough was formed. It was then packed in a specially constructed disc-shaped steel mould to produce a disc specimen $(3.8 \mathrm{~mm}$ diameter and $1.0 \mathrm{~mm}$ thickness). After packing the mould, it was allowed to stand for 13 minutes then placed in a pressure curing unit and cured at $55^{\circ} \mathrm{C}$ and 2 bar pressure for 15 minutes.

\section{High Performance Liquid Chromatography} (HPLC)

Chemicals, reagents and standards

Acetonitril of HPLC-quality, hydrochloric acid and sodium citrate of analytical grade were all purchased from GCC (England). The standard material of the drugs, Fluconazole and Chlorhexidine, were purchased from Cadilla pharmaceuticals (Ahmad-Abad, India). The internal standard pmethyl phenol was purchased from ACROS (Geel, Belgium).

HPLC- Apparatus and working conditions

The analysis was carried out on an isocratic HPLC-apparatus (Figure 2) which consists of the following parts: HPLC pump type GBC (Australia), model LC 1110; HPLC injector type Rheodyne 7125 (USA); HPLC-UV-Vis detector type GBC (Austra(ia), model LC 1205; Integrator type Spectra Physics model LC 4290.

The HPLC apparatus was operated under the following working conditions:

Eluent: acetonitril / $0.01 \mathrm{M}$ phosphate buffer (25:75\%); Eluent flow rate: $1.0 \mathrm{ml} / \mathrm{min}$; Injection volume: $20 \mu \mathrm{l}$; Column: BDS - C18 $(25 \mathrm{~cm} \mathrm{x}$ $4.6 \mathrm{~mm}$, particle size $5 \mu \mathrm{m})$; Detector : UV - VIS -Spectrophotometer ( $\lambda: 210 \mathrm{~nm}$, Range: 1.0); integrator chart speed: $0.5 \mathrm{~cm} / \mathrm{min}$, attenuation: 8

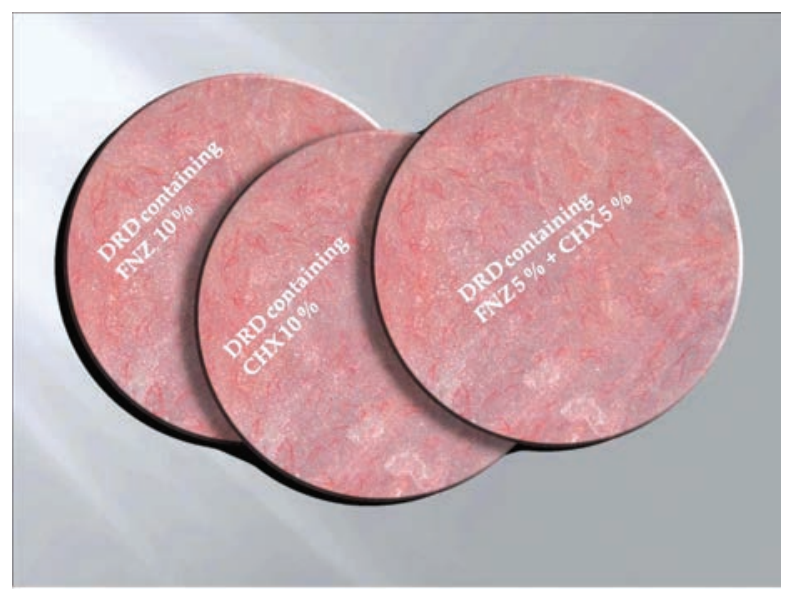

Figure 1. Discs of the three sample groups showing the drug release device (DRD) impregnated with 10\% Fluconazole; 10\% Chlorhexidine, and with a combination of $5 \%$ of each drug. 
Stock standard solutions and working standard solution

A $1000 \mu \mathrm{g} / \mathrm{ml}$ stock standard solution was prepared for each drug by dissolving $10 \mathrm{mg}$ of the drug in $10.0 \mathrm{ml} \mathrm{HPLC}$-water. These prepared solutions were kept in a refrigerator.

The working standard solution for each drug, i.e., Fluconazole or Chlorhexidine, was prepared by mixing $20 \mu \mathrm{g} / \mathrm{ml}$ of the drug with $50 \mu \mathrm{g} / \mathrm{ml}$ of the internal standard $p$-methyl phenol in HPLC-water.

\section{Leaching behavior}

In the first group of samples, five discs, 6.27 g each which contained $10 \% \mathrm{w} / \mathrm{w}$ of the Fluconazole antifungal drug were soaked separately each in $60 \mathrm{ml}$ distilled water. In the second group, five discs, $6.32 \mathrm{~g}$ each which contained $10 \% \mathrm{w} / \mathrm{w}$ of the Chlorhexidine antimicrobial drug were separately soaked each in $60 \mathrm{ml}$ distilled water. In the third group, five discs, $6.25 \mathrm{~g}$ each which contained $5 \% \mathrm{w} / \mathrm{w}$ Fluconazole and 5\% w/w Chlorhexidine were soaked separately each in $60 \mathrm{ml}$ distilled water. The 15 containers which accommodated the samples (five disc specimens for each of the three groups) were left in a thermostatically controlled revolving water bath at $37^{\circ} \mathrm{C}$. A small known amount of the test solution was taken from each container after soaking intervals of 2 hours, 4 hours, 6 hours, 12 hours, 1 day, 2 days, 4 days, 1 week, 2 weeks, 3 weeks and 4 weeks. The distilled water in the 15 containers was renewed at each test interval, in order to simulate the clinical situation regarding the dilution of the thin salivary film at the denture-mucosa interface.

At each time of analysis, the test solution of each sample group was taken and mixed with

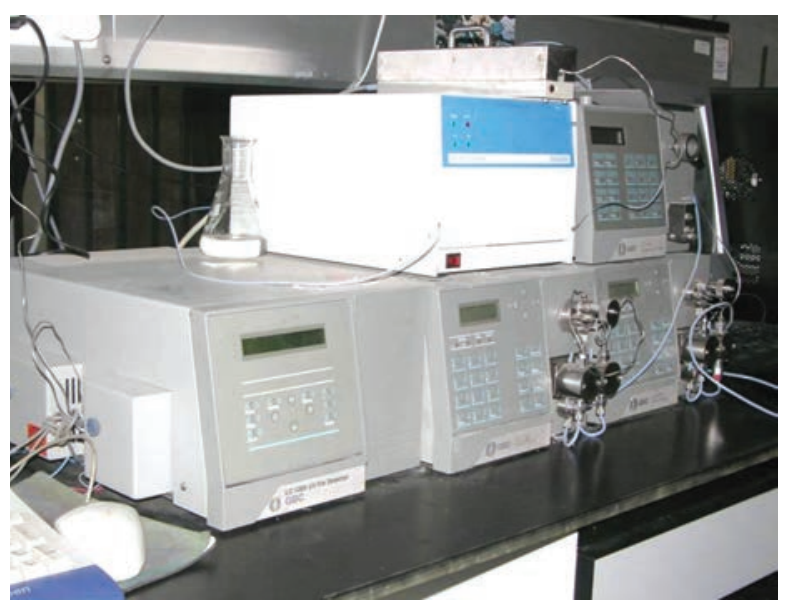

Figure 2. The High Performance Liquid Chromatography (HPLC) apparatus employed in the present investigation. aqueous solution of the internal standard of the corresponding sample, so that its concentration in the final volume $(5 \mathrm{ml}$ ) becomes $50 \mu \mathrm{g} / \mathrm{ml}$. $20 \mu \mathrm{l}$ of this mixture solution was injected onto the HPLCcolumn under the above mentioned conditions.

\section{Qualitative and quantitative analysis}

A representative chromatogram of a standard mixture of Fluconazole with internal standard is shown in Figure 3 and that for Chlorhexidine in Figure 4. A representative chromatogram of the standard mixture which combined Fluconazole and Chlorhexidine is shown in Figure 5.

The qualitative identification of each drug peak was performed through comparing the relative retention time (RRT) of each drug, with respect to the internal standard $p$-methyl phenol in the real sample to those (RRT) in the chromatogram of the standard mixture of the corresponding drug, which in this case 3.668 min for Fluconazole and 8.020 min for Chlorhexidine (Figure 5).

The quantitative determination was performed by using the relative peak areas (RPA) and the relative concentrations (RC).

\section{Microbiological investigation}

Strains and media

A strain of Candida albicans was isolated from an in-patient hospitalized at the Jordan University Hospital and was confirmed by biochemical tests. A reference strain of Candida albicans ATCC90028 was used as a control. Until testing, the yeasts were kept frozen in Brain-Heart broth (Difco Laboratories, Detroit, MI, USA) with $5 \%$ glycerol. For each experiment, the strains were sub-cultured twice on Saborauds agar (Difco) for $24 \mathrm{~h}$ at $35^{\circ} \mathrm{C}$

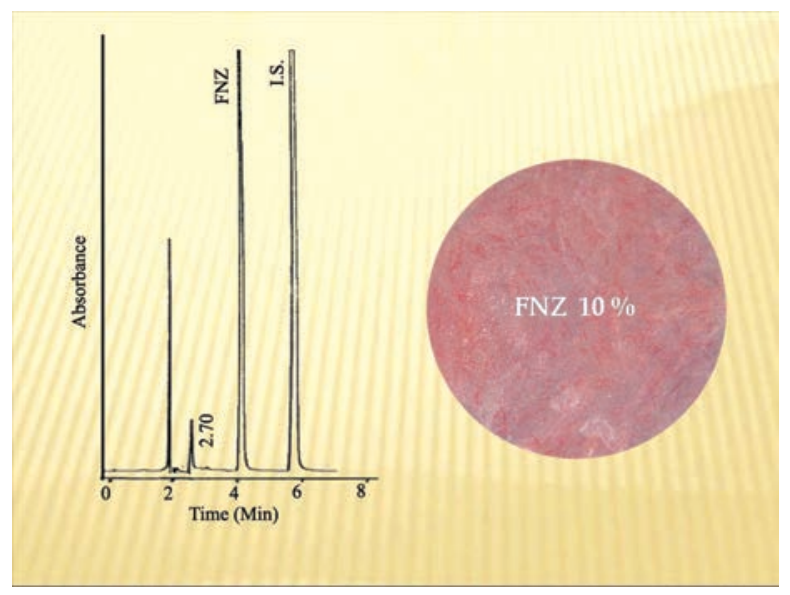

Figure 3. A representative chromatogram of a standard mixture of Fluconazole with the internal standard. 
to ensure viability and purity. The inoculum suspension was prepared by picking five colonies of at least $1 \mathrm{~mm}$ in diameter and suspending them in $5 \mathrm{ml}$ of sterile saline solution $(0.85 \%)$.

\section{The well diffusion test}

This test was performed using Saborauds agar. The inoculum used was prepared using the yeasts from a 24-hour culture on Saborauds agar, a suspension was made in a sterile saline solution $(0.85 \%)$. The turbidity of the suspension was adjusted with a spectrophotometer at $530 \mathrm{~nm}$ to obtain a final concentration to match that of a 0.5 McFarland standard $\left(0.5-2.5 \times 10^{3}\right)$. The inoculated agar was poured into the assay plate $(9 \mathrm{~cm}$ in diameter), and allowed to cool down on a leveled surface. Once the medium had solidified "wells" each $4 \mathrm{~mm}$ in diameter, were cut out of the agar (Figure 7), and $20 \mu \mathrm{l}$ of the eluates from Fluconazole (10\%), Chlorhexidine

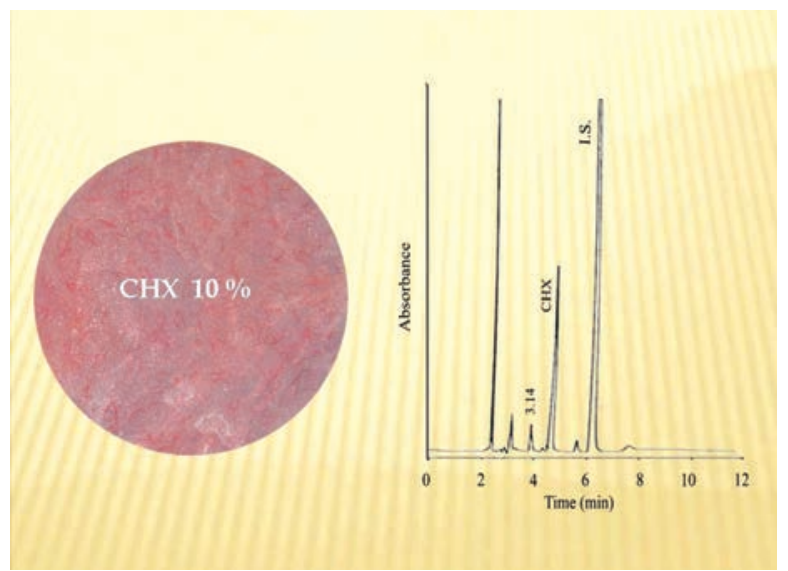

Figure 4. A representative chromatogram of a standard mixture of Chlorhexidine with the internal standard.

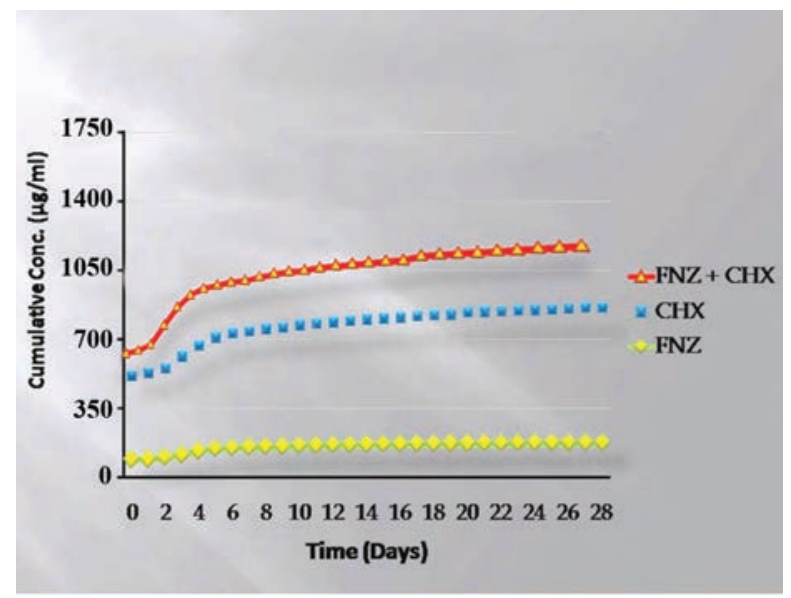

Figure 6. Line diagram plotting the cumulative drug conc $(\mu \mathrm{g} /$ $\mathrm{ml}$ ) versus time (days) for $\mathrm{FNZ}, \mathrm{CHX}$, and $\mathrm{FNZ}+\mathrm{CHX}$ combination, demonstrating the sustained release of the drugs over the four weeks test period.
$(10 \%)$, and the combination drug $(5 \%$ Fluconazole + $5 \%$ Chlorhexidinel were placed each into a well in separate assay plates. Control cups containing the Drug Release Device (DRD) "Control” alone were also included in each assay plate (Figures 8-10). The plates were incubated at $35^{\circ} \mathrm{C}$ for 72 hours.

The absence of growth of Candida albicans, demonstrated by the occurrence of growth inhibition zone around the wells that contained the DRD supplemented with the drugs was interpreted as antifungal activity of the drugs. The antifungal efficacy of each drug was expressed by measurement of the diameter of the inhibition zone, using "pbi Readbiotic" (pbi international, Milano, Italy) measuring device, present around the well which accommodated the DRD that contained the specific drug.

Each experiment was carried out five times for each antifungal drug doped-DRD and was correlated against the controls.

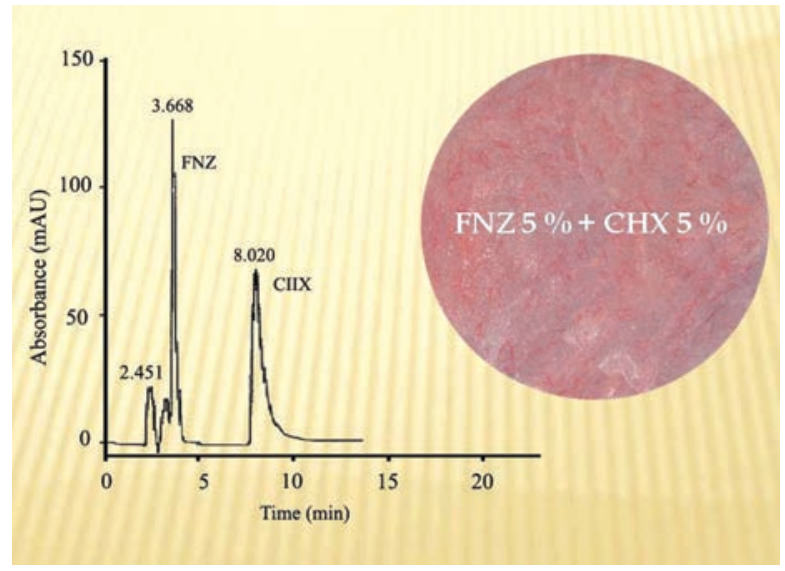

Figure 5. A representative chromatogram of a standard mixture combining Fluconazole and Chlorhexidine.

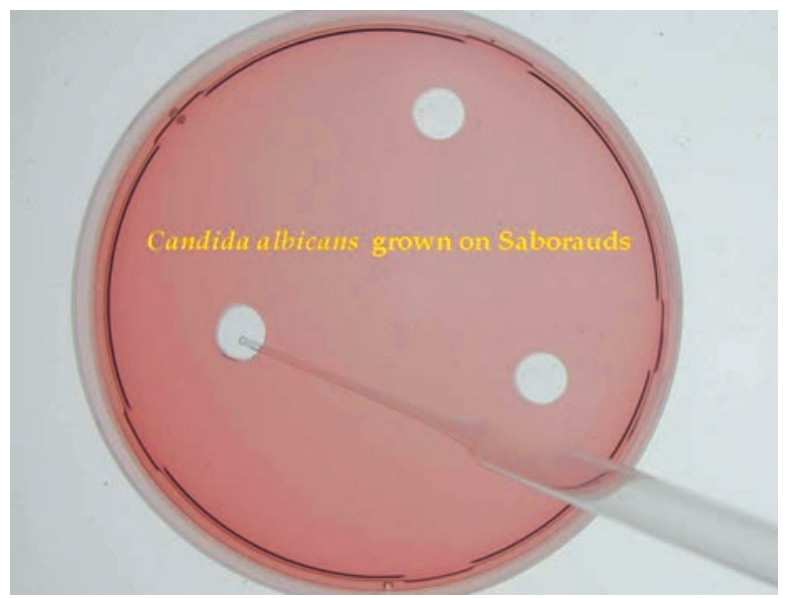

Figure 7. Saborauds culture medium inoculated with Candida albicans. Note the three wells prepared to accommodate the eluates from Fluconazole (10\%), Chlorhexidine (10\%), and the combination drug ( $5 \%$ Fluconazole $+5 \%$ Chlorhexidine) discs soaked in distilled water at mouth temperature.

\section{October 2009 - Vol.3}




\section{Statistical analysis}

Statistical Package for Social Sciences software (SPSS, version 16.0, Inc., Chicago, IL, USA) was employed in the analysis of the various data sets. The differences in the release behavior of Fluconazole, chlorhexidine, and the combination release were determined by Kruskal-Wallis test. The differences in diameter values of the Inhibition Zones of Candida albicans growth produced by Fluconazole, Chlorhexidine, and the combination of the two drugs after 24 hours incubation were determined using the paired-sample t-test. All statistical tests were performed at 0.05 level of confidence.

\section{RESULTS}

Drug release device (DRD)

At the outset, the incorporation with the room temperature polymerized poly (methyl methacrylate) acrylic resin (PMMA) of any of the two em-

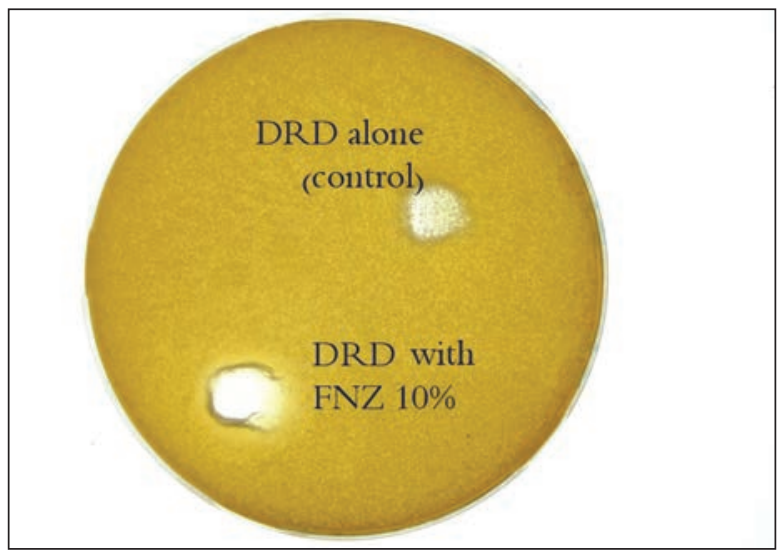

Figure 8. Demonstrating the zone of inhibition of the Candida albicans growth around the well that contained the DRD with $10 \%$ Fluconazole antifungal drug. Note the absence of an inhibition zone around the well that contained the control, i.e., the DRD alone.

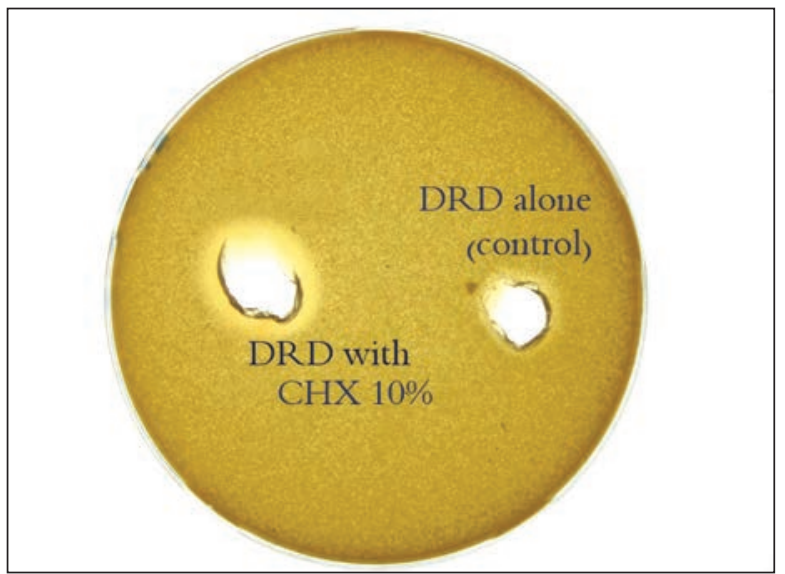

Figure 9. Demonstrating the zone of inhibition of the Candida albicans growth around the well that contained the DRD with $10 \%$ Chlorhexidine antifungal drug. Note the absence of an inhibition zone around the well that contained the control, i.e., the DRD alone. ployed antifungal drugs did not have any untoward effect on the polymerization reaction of the PMMA or on the preparation of the test sample.

\section{HPLC analysis of the drug release}

The antifungal drugs contained in the disc specimens, namely, Fluconazole (10\%), Chlorhexidine $(10 \%)$, and the combination of the two drugs (5\% Fluconazole and 5\% Chlorhexidine) demonstrated an initial high rate of elution from the PMMA reservoir (DRD) during the first 2-7 days followed by a controlled-elution process of sustained release that continued throughout the 28 days test period (Table 1, Figure 6). In general, although the two individual drugs as well as their combination revealed a comparable elution profile but in comparison, Chlorhexidine displayed a substantially higher release rate than Fluconazole $(\mathrm{P}<.05)$, attaining a concentration of up to $606 \mu \mathrm{g} /$ $\mathrm{ml}$ which is equivalent to $36.4 \mathrm{mg}$ of the drug released after one day compared to $128 \mu \mathrm{g} / \mathrm{ml}$ of Fluconazole which is equivalent to $7.7 \mathrm{mg}$ of the drug released during the same period. The rate of release from the samples that combined the two antifungal drugs was significantly higher than that of the individual drugs ( $P=0.002)$. It combined the release rates of the two drugs, and attained a remarkably high released concentration $(734 \mu \mathrm{g} / \mathrm{ml})$ which was equivalent to $44.1 \mathrm{mg}$ of the combined drugs after only one day of exposure distilled water.

\section{Microbiology}

The percolate from the DRD that contained $10 \%$ Fluconazole and that which contained $10 \%$ Chlorhexidine and the DRD that contained a com

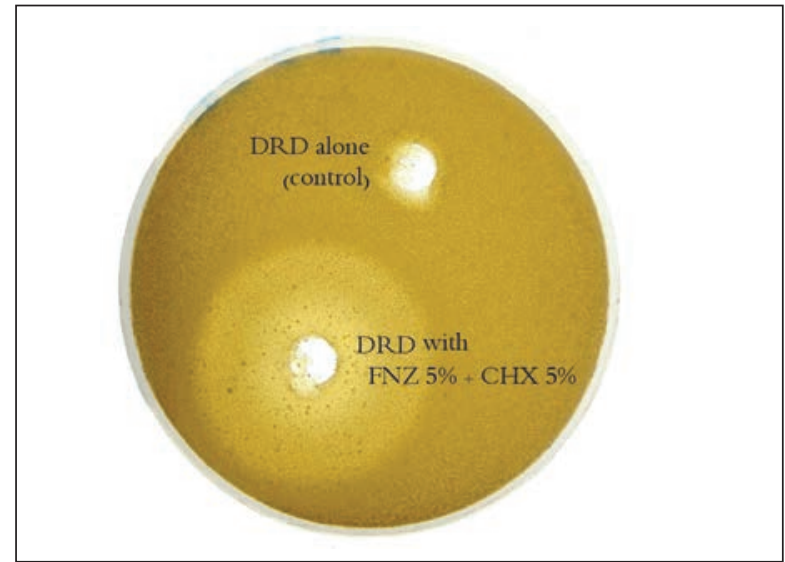

Figure 10. Demonstrating a remarkably wide zone of inhibition of the Candida albicans growth around the well that contained the DRD with combined antifungal drugs, 5\% Fluconazole and $5 \%$ Chlorhexidine. Note the absence of an inhibition zone around the well that contained the control, i.e., the DRD alone. 
bination of $5 \%$ of each antifungal drug showed a clear antifungal activity. This was demonstrated by the occurrence of zones of inhibition of Candida albicans growth around the wells that contained the DRDs with the drugs compared with the controls, i.e., DRD alone (Figures 8-10). However, the three types of DRDs varied in their antifungal potential, with DRD containing Chlorhexidine showing a significantly higher antifungal potential than that containing Fluconazole $(\mathrm{P}<.05)$. The most effective antifungal activity was demonstrated by the DRD that contained the combined drugs ( $P=0.002)$ (Table 2, Figure 11).

The antifungal drugs, Chlorhexidine, Fluconazole and the combination of the two drugs, contained in the DRDs continued to display the same ranking of antifungal potential throughout the 28

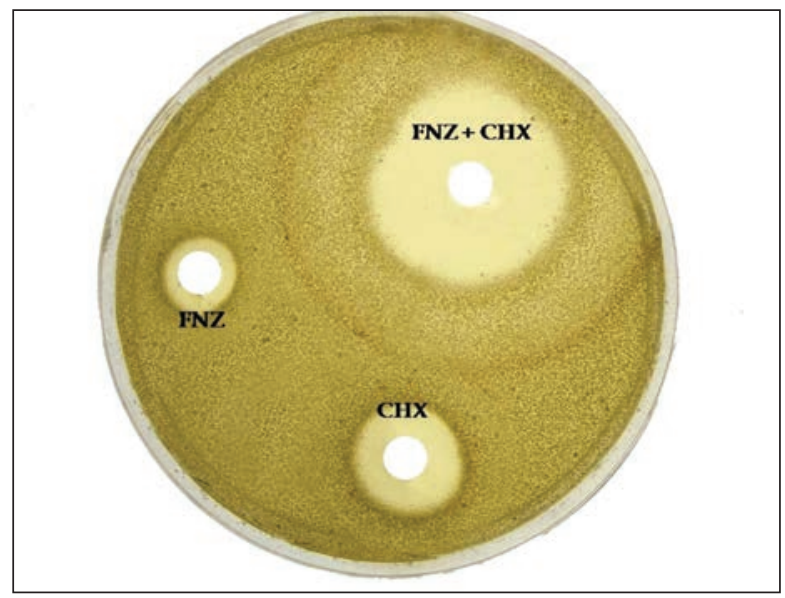

Figure 11. Comparing the zones of inhibition of the Candida albicans growth around the wells that contained the antifungal drugs. Note the remarkably wide inhibition and dilution zones around the well accommodating the combined Fluconazole and Chlorhexidine indicating a synergistic effect of the two antifungal drugs. days test period. This was demonstrated by the plot of the width of the inhibition zone of Candida albicans growth versus time (Figure 12).

\section{DISCUSSION}

The release of Fluconazole and/or Chlorhexidine from the poly (methyl methacrylate), drug release devices, to distilled water indicated that polymerization of the PMMA acrylic resin did not adversely affect the two antifungal drugs nor did doping the PMMA acrylic resin with Fluconazole or Chlorhexidine alter the diffusion characteristics of the resin. This finding is in agreement with previously reported studies that employed polymers for delivering Chlorhexidine. 9,10

Elution of Fluconazole antifungal drug from auto-polymerized acrylic resin specimens doped

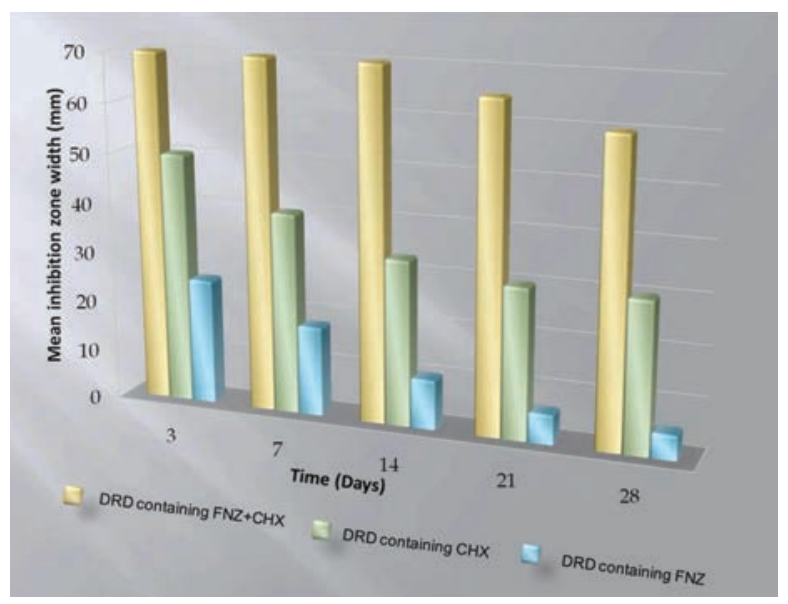

Figure 12. A histogram plotting the width of the inhibition zones of the Candidal growth affected by Fluconazole, Chlorhexidine, and the combination of the two antifungal drugs over the four weeks test period.

Table 1. Mean concentration $(\mu \mathrm{g} / \mathrm{ml}$ ) and amount $(\mathrm{mg})$ values $(\eta=5)$ of the antifungal drugs released from the autopolymerized acrylic discs into distilled water at weekly intervals for the 28 days test period. (Different upper case or lower case alphabets in rows denote significant difference at $\mathrm{P}=0.002$ )

\begin{tabular}{lcccccc}
\hline Time & DRD with10\% Fluconazole & \multicolumn{2}{c}{ DRD with $10 \%$ Chlorhexidine } & \multicolumn{2}{c}{$\begin{array}{c}\text { DRD with 5\% Fluconazole \& } \\
\text { 5\% Chlorhexidine }\end{array}$} \\
\hline \multirow{2}{*}{ (days) } & $\begin{array}{c}\text { Released } \\
\text { conc. }\end{array}$ & $\begin{array}{c}\text { Released } \\
\text { amount }\end{array}$ & $\begin{array}{c}\text { Released } \\
\text { conc. }\end{array}$ & $\begin{array}{c}\text { Released } \\
\text { amount }\end{array}$ & $\begin{array}{c}\text { Released } \\
\text { conc. }\end{array}$ & $\begin{array}{c}\text { Released } \\
\text { amount }\end{array}$ \\
\hline & $(\mu \mathrm{g} / \mathrm{ml})$ & $(\mathrm{mg})$ & $(\mu \mathrm{g} / \mathrm{ml})$ & $(\mathrm{mg})$ & $(\mu \mathrm{g} / \mathrm{ml})$ & $(\mathrm{mg})$ \\
\hline 1 & $128^{\mathrm{A}}$ & $7.68^{\mathrm{a}}$ & $606^{\mathrm{B}}$ & $36.4^{\mathrm{b}}$ & $734^{\mathrm{C}}$ & $44.1^{\mathrm{C}}$ \\
\hline 7 & $202^{\mathrm{A}}$ & $12.12^{\mathrm{a}}$ & $803^{\mathrm{B}}$ & $48.2^{\mathrm{b}}$ & $1005^{\mathrm{C}}$ & $60.3^{\mathrm{c}}$ \\
\hline 14 & $229^{\mathrm{A}}$ & $13.74^{\mathrm{a}}$ & $885^{\mathrm{B}}$ & $53.1^{\mathrm{b}}$ & $1114^{\mathrm{C}}$ & $66.8^{\mathrm{c}}$ \\
\hline 21 & $242^{\mathrm{A}}$ & $14.52^{\mathrm{a}}$ & $946^{\mathrm{B}}$ & $56.8^{\mathrm{b}}$ & $1188^{\mathrm{C}}$ & $71.3^{\mathrm{c}}$ \\
\hline 28 & $249^{\mathrm{A}}$ & $15.00^{\mathrm{a}}$ & $974^{\mathrm{B}}$ & $58.4^{\mathrm{b}}$ & $1223^{\mathrm{C}}$ & $73.4^{\mathrm{c}}$ \\
\hline
\end{tabular}


by Fluconazole demonstrated a relatively high initial release of the drug into distilled water during the first four days. A similar diffusion profile was demonstrated by Chlorhexidine. However, the diffusion gradient of Chlorhexidine was significantly higher than that of Fluconazole.

For both drugs, following the initial elution at a high rate, the release of the antifungal drugs demonstrated a slower and steadier diffusion for up to twenty eight days. The change in the rate of drug release is attributed to the fact that the leaching behavior of Fluconazole and Chlorhexidine into distilled water is governed by a concentration dependent diffusion process. ${ }^{10}$ Although the two antifungal drugs demonstrated somewhat similar general elution behavior, the rate of release, however, was substantially higher for Chlorhexidine than that of Fluconazole. This was indicated by the release of higher concentrations of Chlorhexidine into distilled water throughout the twenty eight days test period compared to Fluconazole which showed release of significantly lower concentrations to distilled water.

Similar elution profile was reported for residual un-polymerized methyl methacrylate monomer from the same auto-polymerizing acrylic resin when exposed to both distilled water and artificial saliva at mouth temperature. ${ }^{11,12}$

The results of the present investigation highlighted the remarkable release behavior of the combined antifungal drugs (Fluconazole and Chlorhexidine $5 \% \mathrm{w} / \mathrm{w}$ each) contained within the PMMA DRD. The diffusion profile revealed by the drug combination DRD demonstrated significantly higher concentrations of the released drugs than the released concentration of either drug individually. The enhanced drug release behavior demonstrated by the drug combination DRDs was consistently high at the outset and continued to be significantly improved throughout the four weeks test period.

It has been clearly established that methacrylate-based polymers absorb up to $30 \%$ water de- pending on the osmolarity of the external solution ${ }^{9}$ or the formulation of the particular polymer. ${ }^{9-11}$ The mechanism of elution seemed to consist of two phases, a rapid linear behavior obeying Fick's law, followed by the development of discrete clusters of the immersion liquid of an un-identified osmotic activity. ${ }^{10}$

In the presence of the antifungal drugs, Chlorhexidine and/or Fluconazole, the rapid elution phase probably indicates surface release process. The subsequent slow phase of sustained release may be the result of complex processes involving formation of fluid clusters around the drug molecules and the interaction of these clusters with the mechanism of fluid absorption of the acrylic resin. Similar behavior has been reported for the release into distilled water but of a different drug from a methacrylate-based polymeric system. ${ }^{\text {? }}$

The elution behavior of Chlorhexidine and/or Fluconazole may also be enhanced by crazes and surface porosity, formed in the brittle poly (methyl methacrylate) by the osmotic forces consequent on the inclusion of the antifungal drug. This is consistent with previously reported finding of a study that used the same polymeric system for delivery of hydrocortisone. ${ }^{13}$

Having established that a Chlorhexidine and/ or Fluconazole-supplemented polymeric devices do release the antifungal drugs in controlled concentrations for up to four weeks, it became essential to investigate whether the concentrations of the released drugs were high enough to affect an antifungal activity upon the growth of Candida albicans which is the common pathogen that causes denture-induced stomatitis lesions. This entailed the microbiological investigation conducted in this study which ascertained that the released concentrations of the antifungal drugs did induce antifungal effect against Candida albicans by inhibiting its growth in Saborauds culture for the entire four weeks test period.

Table 2. Diameter of the inhibition zones of Candida albicans growth produced by Fluconazole, Chlorhexidine, and the combination of Fluconazole and Chlorhexidine after incubation. Each value is the mean diameter of five duplicate trials and was corrected with the control cups. A score of $10 \mathrm{~mm}$ or less was considered insignificant.

\begin{tabular}{lc}
\hline Antifungal Drug & Zone of inhibition in $(\mathrm{mm})$ \\
\hline Fluconazole $10 \%$ & 20 \\
Chlorhexidine $10 \%$ & 25 \\
Fluconazole $5 \%+$ Chlorhexidine $5 \%$ & 55 \\
\hline
\end{tabular}


These findings confirmed those of an earlier study ${ }^{14}$ which also showed that Chlorhexidine diffuses out of an auto-polymerized acrylic resin in fungicidal concentrations over a period of at least three weeks.

The results of the present investigation indicated that Chlorhexidine-supplemented DRD had a more powerful antifungal effect, proved by its capacity of inhibiting the growth of Candida albicans, than that of Fluconazole contained DRD. The results also indicated that the DRD which was supplemented by a combination of the two drugs in a $5 \%$ concentration of each had the most powerful antifungal potential against the growth of Candida albicans, thus indicating a possible synergistic effect of the two drugs.

This is an interesting finding since it encourages the use of combinations of antifungal drugs in low concentrations, thus reducing the chance of developing an allergic reaction to the drugs by the host, yet possessing a substantially high antifungal potential.

In the clinical context, the cumulative nature of Chlorhexidine and Fluconazole release into the surrounding fluid media helps saturate the salivary film which bathe the tissue surface of a denture base with a continuous release bouts of the antifungal drugs.

\section{CONCLUSIONS}

Within the limitations of the present investigation incurred by employing only one resin system for the intraoral delivery of antifungal drugs; and by evaluating the efficacy of the drugs upon the growth of only one candidal species, candida albicans, rather than a wider range of pathogens. The following conclusions, however, may be attained:

- Fluconazole, chlorhexidine and a combination of the two antifungal drugs can be successfully incorporated with auto-polymerized poly (methyl methacrylate)

- These drugs leach out of the polymer into the surrounding fluid medium in an environment similar to that of the oral cavity with respect to humidity and temperature.

- A sustained incremental elution of the antifungal drugs from the doped-polymeric denture base maintains a gradually increasing concentration of the drugs at the exact site of pathology where Candidal infection lesions of the tissue bed of the maxillary denture are usually found.
- The results of the microbiological investigation confirmed the antifungal efficacy of the drugsupplemented delivery systems and favored the use of polymeric systems doped with a combination of antifungal drugs in small concentrations.

Future investigations should be carried out to evaluate the antifungal efficacy of the suggested system against a wider range of pathogens from a higher number of clinical isolates. Similar in vitro studies employing different polymeric systems for a sustained intraoral release of antifungal drugs should be encouraged. Clinical studies are essential in order to test the efficacy of the suggested drug-delivery system, before implementing the new dosage form of antifungal drugs for the treatment of denture-associated oral Candidiasis.

\section{REFERENCES}

1. Budtz-Jørgensen $E$. The significance of Candida albicans in denture stomatitis. Scand J Dent Res 1974;82:5-51.

2. Budtz-Jørgensen E, Theilade E, Theilade J. Regional variations in viable bacterial and yeast counts of 1-week-old denture plaque in denture stomatitis. Scand J Dent Res 1983;91:288-295.

3. Pollack B, Buck IF, Kalnius J. An oral syndrome complicating pschopharmacotherapy: study II. Am J Psychiatry 1964;121:384-386.

4. Budtz-Jørgensen E, Theilade E, Theilade J. Quantitative relationship between yeasts and bacteria in denture-induced stomatitis. Scand J Dent Res 1983;91:134-142.

5. Bergendal T, Holmberg K, Nord CE. Yeast colonization in the oral cavity and feces in patients with denture stomatitis. Acta Odontol Scand 1979;37:37-45.

6. Brook IM, van Noort R. Controlled delivery of drugs. Br Dent $J$ 1984;157:11-15.

7. Garcia CR, Siquerios A, Benet LZ. Oral controlled release preparations. Pharm Acta Helv 1978;53:99-109.

8. Paul DR. Polymers in controlled release technology. American Chemical Society Symp. 1976; Series No. 33 Controlled release polymeric formulations, 1-3.

9. Budtz-Jorgensen E, Lombardi T. Antifungal therapy in the oral cavity. Periodontol 2000 1996;10:89-106.

10. Dar-Odeh NS, Shehabi AA. Oral candidosis in patients with removable dentures. Mycoses 2003;46: 187-191.

11. White TC, Marr KA, Bowden RA. Clinical, cellular, and molecular factors that contribute to antifungal drug resistance. Clin Microbiol Rev 1998;11:382-402. 
12. Johnson EM, Warnock DW, Luker J, Porter SR, Scully C. Emergence of azole drug resistance in Candida species from HIV-infected patients receiving prolonged fluconazole therapy for oral candidosis. $J$ Antimicrob Chemother 1995;35:103-114.

13. Epstein JB. Antifungal therapy in oropharyngeal mycotic infections. Oral Surg Oral Med Oral Pathol 1990;69:32-41.

14. Ellepola AN, Samaranayake LP. Oral candidal infections and antimycotics. Crit Rev Oral Biol Med 2000;11:172-198.

15. Samaranayake LP, McCourtie J, MacFarlane TW. Factors affecting the in-vitro adherence of Candida albicans to acrylic surfaces. Arch Oral Biol 1980;25:611-615.

16. Budtz-Jorgensen E, Carlino P. A miconazole lacquer in the treatment of Candida-associated denture stomatitis. Mycoses 1994;37:131-135.

17. Nikawa H, Yamamoto T, Hamada T, Rahardjo MB, Murata $\mathrm{H}$, Nakanoda S. Antifungal effect of zeolite-incorporated tissue conditioner against Candida albicans growth and/or acid production. J Oral Rehabil 1997;24:350-357.

18. Douglas WH. Dental materials as carriers for therapy. Dent Update 1977;4:395.

19. Douglas WH, Walker DM. Nystatin in denture liners-an alternative treatment of denture stomatitis. $\mathrm{Br}$ Dent $J$ 1973;135:55-59.

20. Schneid TR. An in vitro analysis of a sustained release system for the treatment of denture stomatitis. Spec Care Dentist 1992;12:245-250.

21. Addy M. In vitro studies into the use of denture base and soft liner materials as carriers for drugs in the mouth. $J$ Oral Rehabil 1981;8:131-142.

22. Addy M, Handley R. The effects of the incorporation of chlorhexidine acetate on some physical properties of polymerized and plasticized acrylics. J Oral Rehabil 1981;8:155-163.

23. Garcia CR, Siqueiros A, Benet LZ. Oral controlled release preparations. Pharm Acta Helv 1978;53: 99-109.

24. Mirth DB, Bartkiewicz A, Shern RJ, Little WA. Development and in vitro evaluation of an intra-oral controlled-release delivery system for chlorhexidine. J Dent Res 1989;68:12851288.

25. Riggs PD, Braden M, Patel M. Chlorhexidine release from room temperature polymerising methacrylate systems. Biomaterials 2000;21:345-351.

26. Patel MP, Cruchley AT, Coleman DC, Swai H, Braden M, Williams DM. A polymeric system for the intra-oral delivery of an anti-fungal agent. Biomaterials 2001;22:23192324.

27. Braithwaite A, Smith FJ. Chromatographic methods. London. Blackie Academic and Professional. 1996 